\title{
Traversable wormholes from surgically modified Schwarzschild spacetimes
}

\author{
Matt Visser \\ Theoretical Division \\ T-8, Mail Stop B-285 \\ Los Alamos National Laboratory \\ Los Alamos, New Mexico 87545 \\ Present address: \\ School of Mathematics, Statistics, and Computer Science \\ Victoria University of Wellington, New Zealand \\ matt.visser@mcs.vuw.ac.nz \\ 27 January 1989; $\mathrm{AT}_{\mathrm{E} X-e d}$ October 22, 2018
}

\begin{abstract}
In this paper I present a new class of traversable wormholes. This is done by surgically grafting two Schwarzschild spacetimes together in such a way that no event horizon is permitted to form. This surgery concentrates a nonzero stress-energy on the boundary layer between the two asymptotically flat regions. I shall investigate this stress-energy in detail using the "junction condition" formalism. A feature of the present analysis is that this class of traversable wormholes is sufficiently simple for a (partial) dynamical stability analysis to be carried out. The stability analysis places constraints on the equation of state of the exotic matter that comprises the throat of the wormhole.
\end{abstract}

PACS numbers: 04.20.Jb, 03.70.+k, 04.62.+v, 04.60.-m, 11.10.Kk

Keywords: traversable wormholes, Lorentzian wormholes.

Nuclear Physics B328 (1989) 203-212.

DOI:10.1016/0550-3213(89)90100-4 


\section{Introduction.}

Recently there has occurred a major Renaissance in wormhole physics. While most energies are being foucused on wormholes as possibly significant contributions to quantum gravity, I feel that the analysis of classical traversable wormholes merits serious attention. Analyses of traversable wormholes have recently been presented by Morris and Thorne [1, by Morris, Thorne, and Yurtsever [2], and by the present author [3]. Traversable wormholes are specifically designed so that they may in principle be used by humans to travel between universes (or distant parts of the same universe) without fatal effects on the traveller. A major result of these investigations is that violations of the weak energy hypothesis are guaranteed to occur at the throat of a traversable wormhole.

In this paper I present a new class of traversable wormholes. In an earlier publication, I discussed a class of spherically asymmetric traversable wormholes [3]. In this paper I shall pursue a different topic. I shall adopt the constraint of spherical symmetry, and shall assume that all matter is confined to a thin boundary layer between universes. Thus the models I consider are a subset of the "absurdly benign" wormholes of reference [1]. The models are constructed by by surgically grafting two Schwarzschild spacetimes together in such a way that no event horizon is permitted to form. This surgery (naturally) concentrates a non-zero stress-energy on the boundary layer between the two universes. We shall investigate this stress-energy in detail using the "junction condition" formalism (a.k.a. the "boundary layer" formalism). A major innovation in the present analysis is that these wormholes are sufficiently simple for a (partial) dynamical stability analysis to be carried out. Such a stability analysis was totally unmanageable in the models considered in references [1, 2, 3]. The generalization to traversable wormholes based on surgically modified ReissnerNordström spacetimes is immediate.

It is most illuminating to first construct the class of models to be considered in the static case, ignoring stability questions. Once details of the static case have been spelled out, I shall turn attention to the dynamical analysis of stability. I shall be limited to considering spherically symmetric motions of the wormhole throat. The technical details of this analysis will closely parallel that of Blau, Guendelman, and Guth [4], though they were looking at a totally different physical system.

The static analysis already enforces the presence of "exotic stress-energy" (i.e., violations of the weak energy hypothesis [1, 2, 3]). The stability analysis places constraints on the equation of state of this exotic stress-energy. Suitable candidates for the equation of state of this exotic stress-energy are identified. If the wormhole is to be absolutely stable, (rather than metastable), then the gravitational mass as 
measured at spatial infinity must be non-positive. This behavior is alarming, but not necessarily fatal.

\section{Schwarzschild surgery.}

To construct the wormholes of interest, consider the ordinary Schwarzschild solution to the vacuum Einstein field equations:

$$
d s^{2}=-\left(1-\frac{2 M}{r}\right) d t^{2}+\frac{d r^{2}}{\left(1-\frac{2 M}{r}\right)}+r^{2}\left(d \theta^{2}+\sin ^{2} \theta d \phi^{2}\right) .
$$

I utilize the ordinary Scwarzschild coordinates, and do not maximally extend the manifold, as that would prove to be unprofitable. Now take two copies of this manifold, and remove from them the four-dimensional regions described by $\Omega_{1,2} \equiv\left\{r_{1,2} \leq\right.$ $a \mid a>2 M\}$. One is left with two geodesically incomplete manifolds with boundaries given by the timelike hypersurfaces $\partial \Omega_{1,2} \equiv\left\{r_{1,2}=a \mid a>2 M\right\}$. Now identify these two timelike hypersurfaces (i.e., $\partial \Omega_{1} \equiv \partial \Omega_{2}$ ). The resulting spacetime $\mathscr{M}$ is geodesically complete and possesses two asymptotically flat regions connected by a wormhole. The throat of the wormhole is at $\partial \Omega$. Because $\mathscr{M}$ is piecewise Schwarzschild, the stress-energy tensor is everywhere zero, except at the throat itself. At $\partial \Omega$ one expects the stress-energy tensor to be proportional to a delta function. This situation is made to order for an application of the "junction condition" formalism, also known as the "boundary layer" formalism [4, 5]. The nature and behavior of the stress-energy tensor is the major focus of this paper. Note that the class of traversable wormholes I have just described is a subclass of the "absurdly benign" wormholes of reference 1, obtained in the limit where the thickness of the transition region containing exotic stress-energy shrinks to zero. Finally, note that the condition $a>2 M$ is necessary to prevent the formation of an event horizon.

Since all the stress-energy is concentrated on the throat, the throat may be viewed as behaving like a domain wall between the two universes. The simplest domain wall one can construct is the classical membrane, but this will be shown to be unstable. More generally one may consider a domain wall consisting of a membrane that has some $(2+1)$-dimensional matter trapped on it. Domain walls of this type can in principle possess essentially arbitrary equations of state. We shall use the stability analysis to constrain the equation of state.

I also wish to mention that the analysis soon to be presented generalizes immediately to traversable wormholes based on surgical modifications of the Reissner- 
Nordström spacetime. Merely repeat the above discussion using the metric

$$
d s^{2}=-\left(1-\frac{2 M}{r}+\frac{Q^{2}}{r^{2}}\right) d t^{2}+\frac{d r^{2}}{\left(1-\frac{2 M}{r}+\frac{Q^{2}}{r^{2}}\right)}+r^{2}\left(d \theta^{2}+\sin ^{2} \theta d \phi^{2}\right) .
$$

\section{Junction conditions and the Einstein equations.}

I now briefly review the junction formalism [4, 5]. Consider two four-dimensional spacetimes $\Omega_{1,2}$ with boundaries $\partial \Omega_{1,2}$. We wish to join these manifolds at their boundaries to create a new manifold $\Omega=\Omega_{1} \oplus \Omega_{2}$ that has no boundary. The first junction condition is that the three-dimensional geometries of $\partial \Omega_{1}$ and $\partial \Omega_{2}$ must be the same. (That is, the first fundamental forms of $\partial \Omega_{1}$ and $\partial \Omega_{2}$ must be identical.) This means that we wish the geometry of $\Omega$ to at least be continuous at the junction. However, the metric need not be differentiable at the junction, so the affine connection may be discontinuous there, and the Riemann tensor may possess a delta function singularity there. This statement may be quantified in terms of the second fundamental forms of the boundaries $\partial \Omega_{1,2}$. Let us adopt Riemann normal coordinates at the junction. Let $\eta$ denote a coordinate normal to the junction, with $\eta$ positive in $\Omega_{1}$ and negative in $\Omega_{2}$. The second fundamental forms are then

$$
K_{j}^{i}{ }^{ \pm}=\left.\frac{1}{2} g^{i k} \cdot \frac{\partial g_{k j}}{\partial \eta}\right|_{\eta= \pm 0}
$$

The Ricci tensor at the junction is easily calculated in terms of the discontinuity in the second fundamental forms. Define $\kappa_{i j}=K_{i j}{ }^{+}-K_{i j}{ }^{-}$, then

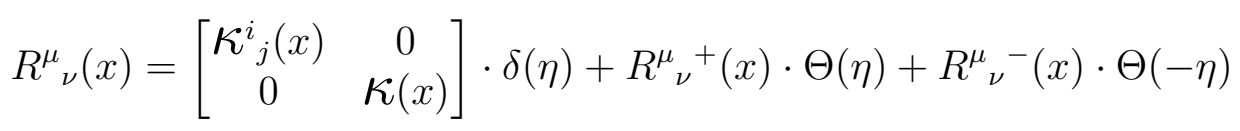

(We adopt the convention that $\Theta(0)=0$.) The stress-energy tensor is

$$
T^{\mu \nu}(x)=S^{\mu \nu}(x) \cdot \delta(\eta)+T^{\mu \nu+}(x) \cdot \Theta(\eta)+T^{\mu \nu-} \cdot \Theta(-\eta) .
$$

Conservation of stress-energy severely constrains the surface stress-energy. It is easy to see that $S^{\eta \eta} \equiv 0 \equiv S^{i \eta}$, so that the only nonzero components of $S$ are the $S^{i}{ }_{j}$. Stress-energy may be exchanged between the surface layer at the junction and its surroundings, subject to the constraint $S^{i j}{ }_{j j}=-T^{i \eta+}+T^{i \eta-}$. Finally the condition of pressure balance reads $\bar{\kappa}_{i j} S^{i j}=T^{\eta \eta+}-T^{\eta \eta-}$, where $\overline{\boldsymbol{K}}_{i j}=\frac{1}{2}\left\{K_{i j}{ }^{+}+K_{i j}{ }^{-}\right\}$. The Einstein field equations lead to an expression for the surface stress-energy tensor

$$
S_{j}^{i}=-\frac{c^{4}}{8 \pi G} \cdot\left[\kappa^{i}{ }_{j}-\delta^{i}{ }_{j} \kappa_{k}^{k}\right] .
$$


This completes the review of the general case. For the spherically symmetric and reflection symmetric case at hand considerable simplifications occur. Firstly, $K^{+}=-K^{-}=\frac{1}{2} \kappa$, while $\bar{\kappa} \equiv 0$. Secondly $T^{ \pm} \equiv 0$, so the pressure balance constraint is vacuous, while for the surface stress-energy $S^{i j}{ }_{\mid j}=0$. Thirdly, spherical symmetry implies that

$$
\kappa_{j}^{i}=\left[\begin{array}{ccc}
\kappa^{\tau}{ }_{\tau} & 0 & 0 \\
0 & \kappa_{\theta}^{\theta} & 0 \\
0 & 0 & \kappa^{\theta}{ }_{\theta}
\end{array}\right]
$$

while the surface stress-energy tensor may be written in terms of the surface energy density $\sigma$ and surface tension $\vartheta$ as

$$
S_{j}^{i}=\left[\begin{array}{ccc}
-\sigma & 0 & 0 \\
0 & -\vartheta & 0 \\
0 & 0 & -\vartheta
\end{array}\right] .
$$

Adopting "Geometrodynamic units" $(G \equiv 1 \equiv c)$, the field equations become

$$
\sigma=-\frac{1}{4 \pi} \cdot \kappa_{\theta}^{\theta} ; \quad \vartheta=-\frac{1}{8 \pi} \cdot\left\{\kappa_{\tau}^{\tau}+\kappa_{\theta}^{\theta}\right\}
$$

This has now reduced the computation of the stress-energy tensor to that of computing the two non-trivial components of the second fundamental form. This is very easy to do in the static case, and still quite manageable if the throat is in motion.

\section{Static wormholes.}

The static case is particularly simple. We note

$$
K_{j}^{i}{ }^{ \pm}=\left.g^{i k} \cdot \frac{\partial g_{k j}}{\partial \eta}\right|_{r=a}=\left.\left.\frac{\partial r}{\partial \eta}\right|_{r=a} \cdot g^{i k} \cdot \frac{\partial g_{k j}}{\partial r}\right|_{r=a .}
$$

Now $\frac{\partial r}{\partial \eta}=\sqrt{1-\frac{2 M}{r}}$, so that

$$
K_{\tau}^{\tau} \pm= \pm \frac{\frac{M}{a^{2}}}{\sqrt{1-\frac{2 M}{a}}} ; \quad K_{\theta}^{\theta} \theta^{ \pm}= \pm \frac{\sqrt{1-\frac{2 M}{a}}}{a} .
$$

Which immediately leads to

$$
\sigma=-\frac{1}{2 \pi a} \cdot \sqrt{1-\frac{2 M}{a}} ; \quad \vartheta=-\frac{1}{4 \pi a} \cdot \frac{1-\frac{M}{a}}{\sqrt{1-\frac{2 M}{a}}}
$$


Note that the energy density is negative. This is just a special case of the defocusing arguments presented in references [1, 2, 3] whereby the presence at the throat of "exotic" matter (matter that violates both the weak energy hypothesis and the averaged weak energy hypothesis) was inferred. While somewhat alarming, should not cause too much consternation. It cannot be strongly enough emphasised that the weak energy hypothesis has been experimentally tested in the laboratory, and has been experimentally shown to be false. The averaged weak energy hypothesis is more subtle to test experimentally, and no conclusive results can presently be drawn. It is not commonly appreciated, but it is in fact true, that the observation of the Casimir effect between parallel plates [6] is sufficient to experimentally disprove the weak energy hypothesis, (and also disprove the strong and dominant energy hypotheses). For analyses of the form of the stress-energy tensor between parallel plates see Gibbons [7] and DeWitt [8]. Further comments along these lines may be found in Roman [9], and in the seminal articles by Morris and Thorne [1], and Morris, Thorne, and Yurtsever [2]. In this regard it is perhaps somewhat embarrassing to realise that the experimental observations disproving the (unaveraged) energy hypotheses predate the formulation of the (unaveraged) energy hypotheses by some 25 years. It is an open question as to whether or not quantum theory satisfies the averaged weak energy hypothesis, so until this question is settled the existence of this class of traversable wormholes should be viewed with some caution. In the meantime, it would appear that the best prospects for a theoretical understanding of exotic stress-energy are within the context of semiclassical quantum gravity. The surface tension is also negative, but this merely implies that we are dealing with a surface pressure, not a tension. It should not be too surprising that a positive pressure is needed to prevent collapse of the wormhole throat.

Two special cases are of immediate interest:

- The classical membrane, described by the three-dimensional generalization of the Nambu-Goto action, satisfies the equation of state $\sigma=\vartheta$. In this case $a=3 M$ and

$$
\sigma=\vartheta=-\frac{1}{2 \pi a} \cdot \frac{1}{\sqrt{3}}
$$

This should be compared with the analysis in reference [3], wherein negative tension classical strings were used to construct spherically asymmetric wormholes with polyhedral throats. When we turn to the dynamical analysis, we shall quickly see that this type of wormhole is dynamically unstable.

- Traceless stress-energy. The case $S_{k}^{k}=0$ (i.e., $\sigma+2 \vartheta=0$ ) is of interest because it describes massless stress-energy confined to the throat. (Such a stressenergy tensor arises from considering the Casimir effect for massless fields, a popular 
way of obtaining exotic stress-energy [1, 2, 3.) Unfortunately, in this case no solution to the Einstein field equations exists. ( $\sigma, \vartheta$ prove to be imaginary.) This result is rather depressing as it indicates that consideration of the Casimir effect associated with massless fields is rather less useful than expected.

Following the earlier discussion, the analysis immediately generalizes. Observe that for a traversable wormhole based on surgical modification of the ReissnerNordström spacetime:

$$
\sigma=-\frac{1}{2 \pi a} \cdot \sqrt{1-\frac{2 M}{a}+\frac{Q^{2}}{a^{2}}} ; \quad \vartheta=-\frac{1}{4 \pi a} \cdot \frac{1-\frac{M}{a}}{\sqrt{1-\frac{2 M}{a}+\frac{Q^{2}}{a^{2}}}} .
$$

\section{Dynamic wormholes.}

To analyse the dynamics of the wormhole, we permit the radius of the throat to become a function of time $a \mapsto a(t)$. Note that by an application of Birkhoff's theorem we can be confident that at large radius [in fact for any $r>a(t)$ ] the geometry will remain that of a piece of Schwarzschild spacetime (or Reissner-Nordström spacetime). In particular, the assumed spherical symmetry is a sufficient condition for us to conclude that there is no gravitational radiation regardless of the behavior of $a(t)$. Let the position of the throat of the wormhole be described by $x^{\mu}(t, \theta, \phi) \equiv(t, a(t), \theta, \phi)$, so that the four velocity of a piece of stress-energy at the throat is:

$$
U^{\mu}=\left(\frac{d t}{d \tau}, \frac{d a}{d \tau}, 0,0\right)=\left(\frac{\sqrt{1-\frac{2 M}{a}+\dot{a}^{2}}}{1-\frac{2 M}{a}}, \quad \dot{a}, \quad 0, \quad 0\right)
$$

The unit normal to $\partial \Omega$ is computed to be

$$
\xi^{\mu}=\left(\frac{\dot{a}}{1-\frac{2 M}{a}}, \sqrt{1-\frac{2 M}{a}+\dot{a}^{2}}, \quad 0,0\right) .
$$

The $\theta \theta$ and $\phi \phi$ components of the second fundamental form are

$$
K_{\theta}^{\theta} \equiv K_{\phi}^{\phi}=\left.\frac{1}{r} \cdot \frac{\partial r}{\partial \eta}\right|_{r=a}=\frac{1}{a} \cdot \sqrt{1-\frac{2 M}{a}+\dot{a}^{2}} .
$$

Evaluating the $\tau \tau$ component of $K$ is more difficult. One may, naturally, proceed 
via brute force. It is more instructive to present a short digression. We note that

$$
\begin{aligned}
K_{\tau}^{\tau}=-K_{\tau \tau} & =\xi_{\tau ; \tau} \\
& =-U^{\mu} U^{\nu} \xi_{\nu ; \mu} \\
& =+U^{\nu} \xi_{\mu} U^{\mu} ; \nu \\
& =\xi_{\mu}\left(U^{\mu}{ }_{; \nu} U^{\nu}\right) \\
& =\xi_{\mu} A^{\nu} .
\end{aligned}
$$

Here $A^{\mu}$ is the four-acceleration of the throat. By the spherical symmetry of the problem, the four-acceleration is proportional to the unit normal $A^{\mu} \equiv A \cdot \xi^{\mu}$, so that $K_{\tau}^{\tau}=A \equiv$ magnitude of the four-acceleration. To evaluate the four-acceleration, utilize the fact that $k^{\mu} \equiv\left(\frac{\partial}{\partial t}\right)^{\mu} \equiv(1,0,0,0)$ is a Killing vector for the underlying Schwarzschild geometry. Note that $k_{\mu}=\left(-\left(1-\frac{2 M}{a}\right), 0,0,0\right)$, so that $\dot{a}=-k_{\mu} \xi^{\mu}$, and $\sqrt{1-\frac{2 M}{a}+\dot{a}^{2}}=-k_{\mu} U^{\mu}$. With considerable inspired guess-work and hindsight, it becomes interesting to evaluate:

$$
\begin{aligned}
\frac{D}{D \tau}\left(k_{\mu} U^{\mu}\right) & =k_{\mu ; \nu} U^{\nu} U^{\mu}+k_{\mu} \frac{D U^{\mu}}{D \tau} \\
& =k_{\mu} A \xi^{\mu} \\
& =-A \dot{a} .
\end{aligned}
$$

On the other hand

$$
\begin{aligned}
\frac{D}{D \tau}\left(k_{\mu} U^{\mu}\right) & =-\frac{D}{D \tau} \sqrt{1-\frac{2 M}{a}+\dot{a}^{2}} \\
& =-\frac{1}{\sqrt{1-\frac{2 M}{a}+\dot{a}^{2}}} \cdot\left(\frac{M}{a^{2}}+\ddot{a}\right) \cdot \dot{a} .
\end{aligned}
$$

Comparing the two calculations, we find that the four-acceleration of the throat is

$$
A=\frac{\left(\ddot{a}+\frac{M}{a^{2}}\right)}{\sqrt{1-\frac{2 M}{a}+\dot{a}^{2}}} .
$$

The Einstein field equations become

$$
\sigma=-\frac{1}{2 \pi a} \cdot \sqrt{1-\frac{2 M}{a}+\dot{a}^{2}} ; \quad \vartheta=-\frac{1}{4 \pi a} \cdot \frac{\left(1-\frac{M}{a}+\dot{a}^{2}+a \ddot{a}\right)}{\sqrt{1-\frac{2 M}{a}+\dot{a}^{2}}}
$$

It is relatively easy to check that equations 5.8 imply the conservation of stress-energy

$$
\dot{\sigma}=-2(\sigma-\vartheta) \frac{\dot{a}}{a}, \quad \text { that is: } \quad \frac{D}{D \tau}\left(\sigma a^{2}\right)=\vartheta \cdot \frac{D}{D \tau}\left(a^{2}\right)
$$


As is usual, there is a redundancy between the Einstein field equations and the covariant conservation of stress-energy. With the field equations for a moving throat in hand, the dynamical stability analysis will prove simple.

\section{Stability Analysis.}

The Einstein equations obtained in the previous section may be recast as the pair

$$
\dot{a}^{2}-\frac{2 M}{a}-(2 \pi \sigma a)^{2}=-1 ; \quad \dot{\sigma}=-2(\sigma-\vartheta) \frac{\dot{a}}{a} .
$$

Consider the classical membrane. The equation of state is $\sigma=\vartheta$, so that $\dot{\sigma} \equiv 0$. It is immediately clear from equation 6.1 that a traversable wormhole built using a classical membrane is dynamically unstable. We need merely observe that the potential in 6.1 is unbounded below. Wormholes of this type either collapse to $a=0$ or blow up to $a=\infty$ depending on the initial conditions. For example, if $a \gg M$ we may write down the approximate solution $a(\tau)=\frac{1}{2 \pi \sigma} \cosh (2 \pi \sigma \tau)$. Even the somewhat outré condition that $M<0$ will only help to stabilize the wormhole against collapse, it will do nothing to prevent the system exploding. It should be noted that since the surface energy density is already negative, the possibility of a negative total gravitational mass is no longer excluded. Since the presence of the wormhole has allowed us to excise the otherwise naked singularity at $r=0$, this geometry does not violate the cosmic censorship hypothesis even for $M<0$. Furthermore, even if $M<0$, one still must require $2 \pi \cdot a^{3 / 2} \cdot \sigma(a) \rightarrow k_{0}<\sqrt{2|M|}$ as $a \rightarrow 0$ in order for the surface density term to not overwhelm the mass term.

More generally note that for $M>0$ the potential near $a=0$ is unbounded below, regardless of the behavior of $\sigma(a)$. Indeed if $a<2 M$, we see that a runaway solution develops with $a \rightarrow 0$ as $\tau \rightarrow \infty$. Physically this is a reflection of the fact that if the throat falls within its own Schwarzschild radius, then the wormhole is doomed. Thus if $M>0$ the best we can hope for is that the wormhole be metastable against collapse to $a=0$.

If we consider the behavior as $a \rightarrow \infty$, we see that the wormhole is stable against explosion if and only if $2 \pi \cdot a \cdot \sigma(a) \rightarrow k_{\infty}<1$. If this condition is violated, the wormhole is at best metastable. This condition on $\sigma(a)$ will be shown to imply a constraint on the equation of state of the domain wall in the region $\sigma \approx 0$. 


\section{Equation of state.}

To constrain the equation of state, we use the fact that $2 \pi \cdot a \cdot \sigma(a) \rightarrow k_{\infty}<1$ as $a \rightarrow \infty$. Since this implies that $\sigma(a) \rightarrow 0$ at spatial infinity, it becomes interesting to expand the equation of state in a Taylor series around $\sigma=0$ :

$$
\vartheta(\sigma)=\vartheta_{0}+k^{2} \sigma+o\left(\sigma^{2}\right) .
$$

From this assumed equation of state, and the conservation of stress-energy, one may calculate $\sigma(a)$. Specifically, ignoring $o\left(\sigma^{2}\right)$ terms

$$
\vartheta_{0}+k^{2} \sigma=\vartheta=\sigma+\frac{1}{2} a \frac{d \sigma}{d a}
$$

This differential equation is easily solved

$$
\sigma(a)=\frac{\vartheta_{0}}{1-k^{2}}+\sigma_{0} \cdot\left(a / a_{0}\right)^{2\left(k^{2}-1\right)} .
$$

By looking at the $a \rightarrow \infty$ behavior we see that $\vartheta_{0}=0$ and $k^{2} \leq \frac{1}{2}$. On the other hand, since equations 5.8 imply that $\sigma$ and $\vartheta$ have the same sign, $k^{2} \geq 0$. Thus

$$
\vartheta(\sigma)=k^{2} \sigma+o\left(\sigma^{2}\right) ; \quad k \in\left[0, \frac{1}{\sqrt{2}}\right] .
$$

In particular, the case $k=0$, (corresponding to negative energy dust), is stable against explosion. Dust is, however, unstable against collapse. To stabilize the wormhole requires either (i) $M=0$ and $k \geq \sqrt{1 / 2}$, or (ii) $M<0$ and $k>1 / 2$, or (iii) $M<0, k=1 / 2$, and $2 \pi \sigma_{0} a_{0}^{3 / 2}<\sqrt{2|M|}$. So for the case $M=0$ we are uniquely led to $k=1 / \sqrt{2}, \vartheta=\frac{1}{2} \sigma, \sigma=\sigma_{0} a_{0} / a$; while for $M<0$ the entire range $k \in(1 / 2,1 / \sqrt{2}]$ is acceptable, corresponding to the range of "semi-soft" equations of state between $\vartheta=\frac{1}{4} \sigma$ and $\vartheta=\frac{1}{2} \sigma$. A slightly more general form of the equation of state, stable against both collapse and explosion, is

$$
\vartheta(\sigma)=k_{+}^{2} \cdot \sigma \cdot \Theta\left(\sigma_{0}-\sigma\right)+k_{-}^{2} \cdot \sigma \cdot \Theta\left(\sigma-\sigma_{0}\right) .
$$

(Note the discontinuity at $\sigma=\sigma_{0}$.) This equation of state leads to a surface density

$$
\sigma(a)=\sigma_{0}\left\{\left(\frac{a}{a_{0}}\right)^{2\left(k_{+}^{2}-1\right)} \cdot \Theta\left(a-a_{0}\right)+\left(\frac{a}{a_{0}}\right)^{2\left(k_{-}^{2}-1\right)} \cdot \Theta\left(a_{0}-a\right)\right\} .
$$

Stability against explosion requires $k_{+} \in\left[0, \frac{1}{\sqrt{2}}\right]$, while stability against collapse requires $k_{-} \in\left[\frac{1}{2}, \infty\right)$. (If $k_{-}=\frac{1}{2}$, then one also requires $2 \pi \sigma_{0} a_{0}^{3 / 2}<\sqrt{2|M|}$.) For 
example, the equation of state $\vartheta(\sigma)=\sigma \cdot \Theta\left(\sigma-\sigma_{0}\right)$ leads to a wormhole that is stable against both explosion and collapse. This example behaves like dust for $\sigma<\sigma_{0}$, and behaves like a classical membrane for $\sigma>\sigma_{0}$.

The conclusions drawn regarding the equation of state depend critically only upon the assumed Taylor series expansion for $\vartheta(\sigma)$ around $\sigma=0$. It is of course possible (though unlikely) that the equation of state be non-analytic at that point, so that no such expansion exists. I have investigated only one example of this type of behavior. Assume the equation of state is

$$
\vartheta(\sigma)=\sigma_{0} \cdot\left(\sigma / \sigma_{0}\right)^{1+(\nu / 2)}
$$

Then conservation of stress energy implies that

$$
\sigma(a)=\sigma_{0} \cdot\left[1+\left(a / a_{0}\right)^{\nu}\right]^{-2 / \nu} .
$$

If $\nu$ is positive, then $\sigma \rightarrow \sigma_{0}\left(a / a_{0}\right)^{-2}$ as $a \rightarrow \infty$, so the wormhole is stable against explosion. Also if $\nu>0$, then $\sigma \rightarrow \sigma_{0}$ as $a \rightarrow 0$, so the wormhole is stable against collapse for $M \leq 0$.

\section{Conclusions.}

In this paper I have described and investigated a new class of traversable wormholes. Because of the simple nature of the geometry a partial stability analysis is possible. As is usual for traversable wormholes, exotic stress-energy (violating the weak energy hypothesis) is present at the throat of the wormhole. Additionally, (global) stability of the wormhole against collapse requires that the gravitational mass of the wormhole as seen by an observer at spatial infinity be non-positive. Constraints are placed on the behavior of the surface energy density as a function of the radius of the throat. These constraints may be transformed into (limited) statements about the equation of state.

The presence of negative energy density and gravitational mass is certainly disturbing but, given our present lack of knowledge, is not immediately fatal. It is encouraging that certain equations of state for the domain wall lead to stable wormholes. It would certainly be of interest to study the hypothesized material comprising the throat in more detail. If nothing else we may yet be able to rule out the existence of traversable wormholes on physical grounds. 


\section{References}

[1] M. S. Morris and K. S. Thorne, "Wormholes in space-time and their use for interstellar travel: A tool for teaching general relativity", Am. J. Phys. 56 (1988) 395.

[2] M. S. Morris, K. S. Thorne and U. Yurtsever, "Wormholes, Time Machines, and the Weak Energy Condition", Phys. Rev. Lett. 61 (1988) 1446.

[3] M. Visser, "Traversable wormholes: Some simple examples", Phys. Rev. D 39 (1989) 3182. (See also arXiv:0809.0907 [gr-qc].)

[4] S. K. Blau, E. I. Guendelman and A. H. Guth, "The Dynamics of False Vacuum Bubbles", Phys. Rev. D 35, 1747 (1987).

[5] C.W. Misner, K.S. Thorne, and J.A. Wheeler, Gravitation, (W.H. Freeman and Company, San Francisco, 1973).

[6] H. B. G. Casimir, "On the Attraction Between Two Perfectly Conducting Plates", Kon. Ned. Akad. Wetensch. Proc. 51 (1948) 793-795;

[Reprinted in various places, see for example: Indag. Math. 10 (1948) 261-263; FRPHA 65 (1987) 342-344; KNAWA,100N3-4 (1997) 61-63.]

[7] G.W. Gibbons, "Stress-Energy in Casimir Effect between parallel plates", in General Relativity: an Einstein Centenary Survey, eds. S.W. Hawking and W. Isreal, page 639 .

[8] B.S. De Witt, "Stress-Energy in Casimir Effect between parallel plates", in General Relativity: an Einstein Centenary Survey, eds. S.W. Hawking and W. Isreal, page 680 .

[9] T. A. Roman, "Quantum Stress Energy Tensors And The Weak Energy Condition", Phys. Rev. D 33 (1986) 3526. 